\title{
No Association Between NRG1 and ErbB4 Genes and Psychopathological Symptoms of Schizophrenia
}

\author{
Sarah Tosato - Martina Zanoni - Chiara Bonetto $\cdot$ Federica Tozzi • \\ Clyde Francks $\cdot$ Elisa Ira $\cdot$ Simona Tomassi $\cdot$ Mariaelena Bertani $\cdot$ \\ Dan Rujescu • Ina Giegling • David St Clair • Michele Tansella • \\ Mirella Ruggeri $\cdot$ Pierandrea Muglia
}

Received: 4 February 2014/Accepted: 25 July 2014/Published online: 21 August 2014

(C) Springer Science+Business Media New York 2014

\begin{abstract}
Neuregulin 1 (NRGl) and v-erb-a erythroblastic leukemia viral oncogene homolog 4 (ErbB4) have been extensively studied in schizophrenia susceptibility because of their pivotal role in key neurodevelopmental processes. One of the reasons for the inconsistencies in results could be the fact that the phenotype investigated has mostly the diagnosis of schizophrenia per se, which is widely heterogeneous, both clinically and biologically. In the present study we tested, in a large cohort of 461 schizophrenia patients recruited in Scotland, whether several SNPs in $N R G 1$ and/or ErbB4 are associated with schizophrenia symptom dimensions as evaluated by the Positive and Negative Syndrome Scale (PANSS). We then followed up nominally significant results in a second cohort of 439 schizophrenia subjects recruited in Germany. Using linear regression, we observed two different groups of polymorphisms in NRG1 gene: one showing a nominal association
\end{abstract}

S. Tosato $(\bowtie) \cdot$ M. Zanoni $\cdot$ C. Bonetto $\cdot$ E. Ira $\cdot$ S. Tomassi . M. Bertani $\cdot$ M. Tansella $\cdot$ M. Ruggeri

Section of Psychiatry, Department of Public Health and

Community Medicine, University of Verona, Policlinico G.B.

Rossi, P.le L.A. Scuro 10, 37134 Verona, Italy

e-mail: sarah.tosato@univr.it

F. Tozzi · C. Francks · P. Muglia

Genetics Division, GlaxoSmithKline, Verona, Italy

C. Francks

Max Planck Institute for Psycholinguistics, Nijmegen,

The Netherlands

D. Rujescu · I. Giegling

Department of Psychiatry, University of Halle, Halle, Germany

D. St Clair

Institute of Medical Sciences, University of Aberdeen,

Aberdeen AB25 2ZD, UK with higher scores of the PANSS positive dimension and the other one with higher scores of the PANSS negative dimension. Regarding ErbB4, a small cluster located in the $5^{\prime}$ end of the gene was detected, showing nominal association mainly with negative, general and total dimensions of the PANSS. These findings suggest that some regions of $N R G 1$ and ErbB4 are functionally involved in biological processes that underlie some of the phenotypic manifestations of schizophrenia. Because of the lack of significant association after correction for multiple testing, our analyses should be considered as exploratory and hypothesis generating for future studies.

Keywords NRG1 - ErbB4 - PANSS · Psychopathology · Schizophrenia

\section{Introduction}

The NRG1 (Neuregulin 1) gene was initially associated with schizophrenia in the Icelandic population (Stefansson et al. 2002), and this association was subsequently replicated in a Scottish case-control study (Stefansson et al. 2003). Since then, a large number of studies in populations of various ethnic backgrounds have reported an association between schizophrenia and NRGI single nucleotide polymorphisms (SNPs) or haplotypes (Gong et al. 2009; Munafo et al. 2008; Tosato et al. 2005). The most consistent and strong association has been detected for a core region including five SNPs and two microsatellites located at the $5^{\prime}$ end of the NRG1 gene (Stefansson et al. 2002). Overall, dozens of SNPs of NRG1 have been associated with schizophrenia, both at 5' (Kukshal et al. 2013; Benzel et al. 2007; Corvin et al. 2004; Hall et al. 2004; Petryshen et al. 2005; Stefansson et al. 2002; Zhao et al. 2004) and $3^{\prime}$ 
regions (Bakker et al. 2004; Benzel et al. 2007; Lachman et al. 2006; Li et al. 2004; Petryshen et al. 2005). Recently, genome-wide association studies (GWAS) for schizophrenia have also reported a nominal association between several SNPs in NRG1 gene and schizophrenia, with $p$ values spanning from $1.59 \times 10^{-5}$ for rs4316112 to $1.38 \times 10^{-3}$ for rs10095694 (Athanasiu et al. 2010; Shi et al. 2009). However, in none of these GWAS, the association survived correction for multiple testing at the threshold proposed for genome-wide significance $\left(p<5 \times 10^{-8}\right)$. Moreover, some meta-analyses have identified several SNPs in the NRG1 gene with overall odds ratios spanning from 0.85 for rs2439272 to 1.14 for rs2954041 (SZGene database http://www.szgene.org/gen eoverview.asp?geneid=311).

This association between $N R G 1$ and schizophrenia thus suggests a role for NRGI as a genetic risk factor for schizophrenia. However, the allelic and population heterogeneity reported across data sets, together with the fact that multiple SNPs and haplotypes have been analyzed, makes it difficult to define $N R G l$ as an unequivocal risk factor for schizophrenia. It is also possible that none of the previously analyzed SNPs represents a suitable proxy for a putative functional polymorphism. In fact, some studies have recently detected no association between schizophrenia and NRG1 gene variants (Crisafulli et al. 2012; Kim et al. 2011; Moon et al. 2011). In order to capture all the existing variability in $N R G l$, the association between $N R G 1$ and schizophrenia can be studied by looking for different tagSNPs or by analyzing hundreds of polymorphisms within the gene.

The multiple reports of an association between schizophrenia and v-erb-a erythroblastic leukemia viral oncogene homolog 4 (ErbB4) (Norton et al. 2006; Silberberg et al. 2006; Benzel et al. 2007), a gene that codes for NRG1 receptor, provide indirect support for the role of NRG1 gene in schizophrenia. In a GWAS, common variants in ErbB4 and its ligand $N R G 1$ were found to be associated with schizophrenia, mostly in African-American subjects (Shi et al. 2009). Some recent meta-analyses suggest the involvement of ErbB4 rs839523 in the susceptibility to schizophrenia in both Caucasian and Asian samples, with an overall odds ratio of 1.01 (SZGene database http://www. szgene.org/geneoverview.asp?geneid=273).

To date, most of the SNPs associated with schizophrenia are noncoding intronic variants, suggesting that the risk ErbB4 SNPs may be functional through splicing regulation or may monitor functional elements within the gene itself (Law et al. 2007). It has therefore been suggested that NRG1 may mediate its effects on schizophrenia susceptibility through functional interactions with ErbB4. It is also possible that a genetic interaction between variants at the two loci increases the genetic risk for schizophrenia
(Norton et al. 2006; Pitcher et al. 2011; Nicodemus et al. 2010). At the same time, however, a recent study has contradicted these results by showing no association between ErbB4 gene and schizophrenia, neither at the SNP nor at the haplotypic levels (Chen et al. 2011).

Overall, the inconsistent findings on the association between $N R G 1, E r b B 4$ and schizophrenia could be due to the fact that the phenotype investigated has been the diagnosis of schizophrenia per se, which is widely heterogeneous, both clinically and biologically (Tosato and Lasalvia 2009; Davidson and McGlashan 1997; Hegarty et al. 1994). Some researchers have suggested that clinical phenotypes would be more closely related than diagnosis to susceptive genes (Li et al. 2013; DeRosse et al. 2012). It has also been suggested that quantitative traits, such as psychopathological dimensions, may lead to a better understanding of the genotype-phenotype relationship (Tao et al. 2006; Wilcox et al. 2002). Unfortunately, only few studies have investigated whether either NRG1 or ErbB4 genes are associated with schizophrenia symptom dimensions, often with contradictory results (Bakker et al. 2004, 2007; Rethelyi et al. 2010; Middle et al. 2010).

In the present study, we tested, in a large cohort of schizophrenia patients recruited in Aberdeen (Scotland), whether tagging SNPs spanning NRG1 and/or ErbB4 were associated with symptom dimensions as evaluated by PANSS. Our aim was to investigate whether genetic variants in NRG1 and ErbB4 genes can influence the severity of clinical symptoms. Moreover, recognizing the importance of replication studies in genetics, we followed up nominally significant results in a second cohort of schizophrenia patients recruited in Munich (Germany).

\section{Materials and Methods}

Sample

The discovery cohort comprised 461 schizophrenia patients (age $24.4 \pm 8.1$ ), all self-identifying as of Scottish or North European ancestry, recruited in Aberdeen, Scotland (Need et al. 2009). The confirmation cohort comprised 439 schizophrenia patients (age $39.2 \pm 10.4$ ), all self-identifying as of German or Central European ancestry, recruited in Munich, Germany (Van den Oord et al. 2006).

Patients were recruited using a consistent clinical protocol. To be enrolled as cases, participants had to fulfill the Diagnostic and Statistical Manual of Mental Disorders, fourth edition (DSM-IV, American Psychiatric Association 1994) criteria for schizophrenia. The diagnosis was validated by Structured Clinical Interview for DSM-IV, SCID I and SCID II (First et al. 1997). All participants were outpatients or clinically stabilized in-patients. Detailed 
medical and psychiatric histories were collected. According to the inclusion criteria, patients were excluded if they: (a) had a history of head injury and/or neurological disease; (b) had a diagnosis of schizoaffective disorder, or a mood disorder unrelated to schizophrenia; (c) were monozygotic twins of cases already included in the study; (d) had been diagnosed as intravenous drug users with dependency during the last 6 months. Further details of the cohorts and protocols are available in previous reports (Van den Oord et al. 2006; Need et al. 2009). After explanation of the procedures and before inclusion in the study, written informed consent was obtained from each subject enrolled in this study. The study was approved by the relevant local ethical committees.

\section{Psychopathology Assessment}

Patients who met inclusion criteria were assessed as soon as possible after they reached a remission of positive psychotic symptoms (i.e., clinical stabilization). Most of the patients were under treatment with antipsychotic medications. Psychopathology was assessed using the Positive and Negative Syndrome Scale (PANSS, Kay et al. 1987) completed with data and observations obtained during the SCID-I interview. The PANSS items yield four scores, regarding: positive symptoms, negative symptoms, general psychopathology and a total score, which is the sum of the first three.

\section{Genotyping}

Venous blood samples $(15 \mathrm{ml})$ were collected in EDTAcontaining tubes. DNA was extracted from blood leukocytes. Two hundreds and sixteen SNPs at the NRG1 locus and 177 SNPs at the ErbB4 locus were selected from the SNPs genotyped through Illumina HumanHap550 Genotyping BeadChip as previously described (Need et al. 2009). A series of quality control (QC) checks and tests of cryptic relatedness was carried out, ultimately excluding a total of 28 participants. A "one percent rule" was also employed, in order to discard from the analysis any SNP for which more $1 \%$ of samples could not be reliably scored. This approach reduced the scope for spurious association while the average success rate of genotyping reached $98.4 \%$ and the concordance rate for duplicate genotyping reached $99.997 \%$ (Need et al. 2009).

\section{Statistical Analysis}

The association between NRG1 and ErbB4 SNPs and psychopathology was tested with a linear regression model using the PLINK software (Purcell et al. 2007) with no covariates. An additive genetic model was used. The null hypothesis H0 was the lack of association between the observed PANSS scores, fitted to a linear equation, and the genotype counts of the minor allele $(0,1,2)$ of each SNP. The $p$ values were corrected for the overall number of analyses conducted to test the PANSS negative, positive and general psychopathology and total scores.

\section{Results}

In the Aberdeen and Munich cohorts, schizophrenia DSMIV subtypes were observed in the following proportions: paranoid 86.2 and $77.6 \%$, disorganized 7.5 and $15.6 \%$, catatonic 2.1 and $2.2 \%$, undifferentiated 4.2 and $4.6 \%$, respectively. PANSS scores were available for 451 subjects in the Aberdeen cohort and for 307 subjects in the Munich sample. PANSS total, positive, negative and general psychopathology scores were $43.46( \pm 12.48)$ and 50.31 $( \pm 15.78), 11.56( \pm 5.24)$ and $13.55( \pm 6.05), 10.50( \pm 3.98)$ and $11.61( \pm 4.68), 21.40( \pm 5.72)$ and $25.15( \pm 7.36)$, respectively. The Munich sample comprised significantly more severe cases than Aberdeen sample, and this could be due to different ascertainment methods at the two sites. While in Munich the participants were chronic outpatients, in Aberdeen they were significantly younger.

Using linear regression and correction for multiple testing, we found that none of the tested SNPs showed a significant association with PANSS psychopathological dimensions, for both $N R G 1$ and ErbB4, in the Aberdeen cohort. Among the initial 216 SNPs investigated in NRG1 locus, 35 SNPs showed a nominal association (before correction for multiple testing) with different psychopathological dimensions in the Aberdeen sample (Table 1).

All these SNPs are located in the region Chr8:31,201,548..33,230,933 (HapMap Data Rel 27 Phase II+III, on NCBI B36 assembly, dbSNP b126). This region comprises the full NRG1 gene and encompasses nearly 400-kbp upstream at its $5^{\prime}$ end and nearly 500-kbp downstream from its $3^{\prime}$ end.

Among the SNPs that showed a nominal association with the positive dimension, the majority was localized between position 31,392,134 and position 31,825,136 at the NRG1 locus (Fig. 1). Among the SNPs that showed a nominal association with the negative dimension, the majority was localized at a different location, between position 32,008,036 and position 32,261,074 (Fig. 1). No such clusters of nominally associated polymorphisms were apparent for either general or total psychopathology dimensions.

Using Haploview (Haploview Software, version 4.2) with data downloaded from HapMap (Version 3, Release 27, reference population CEU, Chr8: from 31,200 to $33,231 \mathrm{kbp}$ ), linkage disequilibrium (LD) patterns for the 
Table 1 All NRG1 SNPs that produced a nominal significant $p$ value $(<0.05$, before correction for multiple testing) with at least one PANSS item scores in the Aberdeen cohort

\begin{tabular}{|c|c|c|c|c|c|}
\hline \multirow[t]{2}{*}{ SNP } & \multirow[t]{2}{*}{ Allele } & \multicolumn{4}{|c|}{ PANSS psychopathological dimensions } \\
\hline & & Positive & Negative & General & Total \\
\hline rs 2681600 & 2 & .0252 & .6592 & .1686 & .2466 \\
\hline rs2681599 & 2 & .0383 & .8186 & .1012 & .1884 \\
\hline rs2716960 & 1 & .0347 & .9248 & .1132 & .1504 \\
\hline rs2716959 & 1 & .0050 & .3693 & .3383 & .3398 \\
\hline rs7813593 & 2 & .5055 & .0712 & .0341 & .0521 \\
\hline rs7828595 & 2 & .0412 & .4991 & .1329 & .2914 \\
\hline rs1476540 & 1 & .0548 & .0420 & .4544 & .9184 \\
\hline$\underline{\text { rs } 1557800^{*}}$ & 1 & .0049 & .1423 & .2332 & .4093 \\
\hline rs10489349 & 1 & .0028 & .5253 & .0104 & .0167 \\
\hline rs986110 & 1 & .0055 & .6656 & .2582 & .1131 \\
\hline rs4281084 & 1 & .0004 & .2112 & .0512 & .0106 \\
\hline rs10113797 & 2 & .0473 & .1412 & .4065 & .1027 \\
\hline rs7463426 & 2 & .0406 & .0186 & .5986 & .0597 \\
\hline rs4733094 & 1 & .0383 & .0274 & .7702 & .0853 \\
\hline rs1503486 & 1 & .2207 & .0492 & .7784 & .1785 \\
\hline rs1354334* & 1 & .0284 & .0880 & .9750 & .1529 \\
\hline rs1566778 & 1 & .0318 & .0824 & .9526 & .1496 \\
\hline rs7827456 & 1 & .5570 & .0233 & .1687 & .1630 \\
\hline rs10954821 & 1 & .6128 & .0122 & .1135 & .1062 \\
\hline rs 13249578 & 1 & .5632 & .0103 & .1374 & .1157 \\
\hline rs11776959 & 1 & .0241 & .3837 & .1561 & .0825 \\
\hline rs9297186 & 1 & .0113 & .8268 & .3345 & .2472 \\
\hline rs17620153 & 1 & .1510 & .1080 & .0197 & .0275 \\
\hline rs1481763 & 2 & .0594 & .0072 & .0081 & .0032 \\
\hline rs4733306 & 1 & .4400 & .0118 & .4060 & .0921 \\
\hline rs 10097263 & 1 & .0485 & .0396 & .1463 & .0306 \\
\hline rs6468090 & 1 & .3179 & .0072 & .2710 & .0510 \\
\hline rs 2200047 & 2 & .3179 & .0072 & .2710 & .0510 \\
\hline rs 10503901 & 1 & .0359 & .3801 & .0119 & .0284 \\
\hline rs11782156 & 1 & .0686 & .2347 & .0349 & .0405 \\
\hline rs16879088 & 1 & .9200 & .0098 & .5390 & .1844 \\
\hline rs4733117 & 2 & .0435 & .1345 & .1354 & .0497 \\
\hline rs 10503902 & 2 & .9430 & .0364 & .9299 & .3470 \\
\hline rs716144 & 2 & .1532 & .0951 & .0243 & .0284 \\
\hline rs4236723 & 2 & .0515 & .6770 & .0396 & .0819 \\
\hline
\end{tabular}

Significant $p$ values $(<0.05$, before correction for multiple testing) are highlighted in bold

* These SNPs have already been found as significantly associated by Petryshen et al. (2005)

investigated SNPs were checked in terms of $r^{2}$. We observed that the two clusters of SNPs mentioned above were not in strong LD (Fig. 2).

Even if our study is essentially a negative study, we followed up our results in the Munich cohort finding that in only a few instances there was a nominal association detected in both of the cohorts. These associations were between one psychopathological dimension and the same polymorphism, with the majority of SNPs showing nominal association only in the Aberdeen cohort but not in the Munich one. In detail, we found that only two SNPs have nominal association with both cohorts. In fact, rs2681599 was associated with the positive PANSS dimension in the Aberdeen sample (uncorrected $p=0.0383$ ), but with the positive and total PANSS score in the Munich sample (uncorrected $p=0.0297$ and 0.0479 , respectively). rs7813593 was associated with the general psychopathology PANSS dimension in the Aberdeen sample (uncorrected $p=0.0341$ ), but with the positive PANSS dimension in the Munich cohort (uncorrected $p=0.0087$ ). There was therefore no notable overlap between significant SNPs in the two cohorts.

Regarding the ErbB4 gene, we found that 21 out of the initially 117 tested SNPs had a nominal association (before correction for multiple testing) with at least one of the four PANSS psychopathological dimensions before correction for multiple testing (Table 2).

All these SNPs were located in the region $\mathrm{Chr}$ : 211,965,043..213,109,527 (HapMap Data Rel 27 Phase II+III, on NCBI B36 assembly, dbSNP b126), which comprised the ErbB4 gene and approximately 50-kbp downstream of its $3^{\prime}$ end and nearly $100 \mathrm{kbp}$ upstream of its $3^{\prime}$ end. The nominally associated SNPs were distributed throughout the region of interest, but notably a small cluster was detected at the $5^{\prime}$ end of the gene (approximately between bp positions $211,847,782$ and $211,934,422$ ). In this region, the SNPs showed nominal associations mainly with negative, general and total dimensions of the PANSS (Fig. 3). Similarly to what was detected for $N R G 1$, using Haploview (Haploview Software, version 4.2) with data downloaded from HapMap (Version 3, Release 27, reference population CEU, Chr2: from 211,800 to 213,110 kbp), we observed that the SNPs of interest were located in a low LD region (Fig. 4).

rs6728697 was the only SNP showing a nominal significant association with all four dimensions of the PANSS in the Aberdeen cohort (uncorrected $p=0.016$ for positive, 0.009 for negative, 0.005 for general and 0.001 for total scores, respectively).

As for $N R G 1$, we followed up our results in the Munich cohort. We found that rs6728697 was the only SNP showing an association (uncorrected $p$ value $=0.016$ in the Aberdeen sample, 0.021 in the Munich sample) with the positive PANSS dimension in both cohorts, before correction for multiple testing. In contrast, all other SNPs showed nominal significant $p$ values $<0.05$ in the Aberdeen but not in the Munich sample. 
SNPs range: $31,201,548 . .33,230,933(2 \mathrm{Mbp})$

NRG1: Chr. 8p12
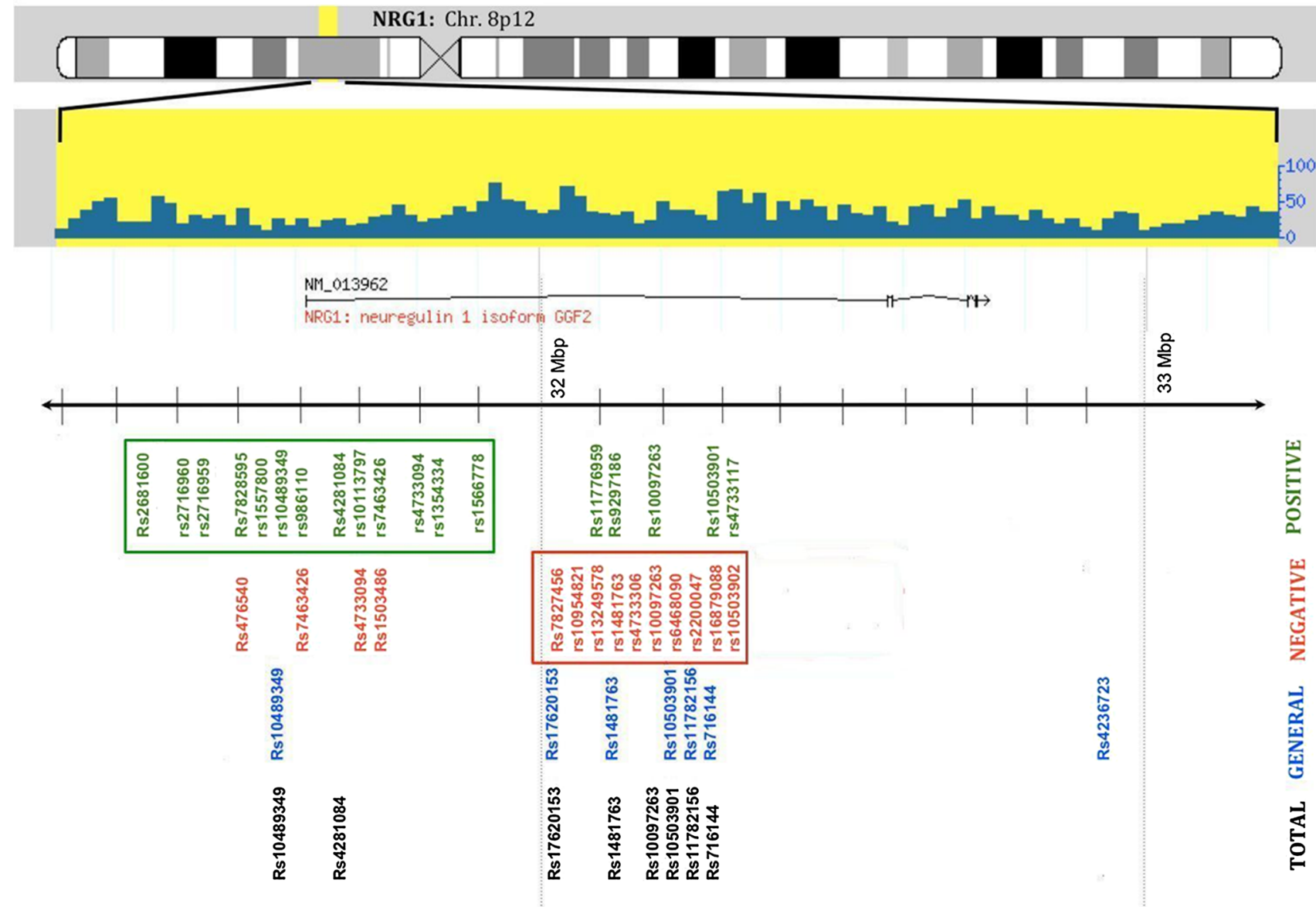

Fig. 1 NRG1 locus along chr 8 and schematic representation of the SNPs that showed a positive correlation with psychopathological symptoms categories (green positive dimension, red negative dimension, blue general dimension, black total dimension) (Color figure online)

\section{Discussion}

To our knowledge, this is the first study testing the relationship between genetic variants in both NRG1 and ErbB4 genes and clinical features in two large cohorts of patients with schizophrenia. The main limitation of this study is the number of variables tested. We performed a substantial number of tests, which means that any Bonferroni correction for multiple testing would have to be large. Consequently, any significant $p$ value should be regarded as nominal.

We also assessed the PANSS cross-sectionally in medicated subjects during the chronic stage of schizophrenia. This could raise the question as to whether the PANSS is a suitable instrument for the identification of real sub-types or categories of schizophrenia (or perhaps if this instrument can capture the intrinsic features of psychosis). Even with this conceptual limitation, the PANSS subscales are commonly used in association studies investigating the potential relationship between polymorphisms and psychopathological profiles (Li et al. 2013; Zhai et al.
2013; Bousman et al. 2013). In our study, chronic schizophrenia subjects were assessed as soon as possible after achieving remission of positive psychotic symptoms (i.e., clinical stabilization). Thus, it is reasonable to think that the psychopathological profile detected captures at least some intrinsic clinical features of psychosis.

Moreover, since patients were medically treated and stable at time of recruitment, it is also possible that our results reflect, at least in part, an effect of pharmacological treatment, partially covering the real impact of genetic variants on schizophrenia symptoms. Our analyses should therefore be considered as exploratory and hypothesisgenerating.

In the present study, some putative "hot-spot" regions in both NRG1 and ErbB4 were identified, in which close SNPs, almost as a cluster, might be those associated with clinical psychopathological dimensions of schizophrenia. Interestingly, we observed that NRG1 SNPs showing a nominal association with schizophrenia had a tendency to group in two distinct regions along the gene length. The first region spans nearly $700 \mathrm{~kb}$ and covers the whole $5^{\prime}$ 


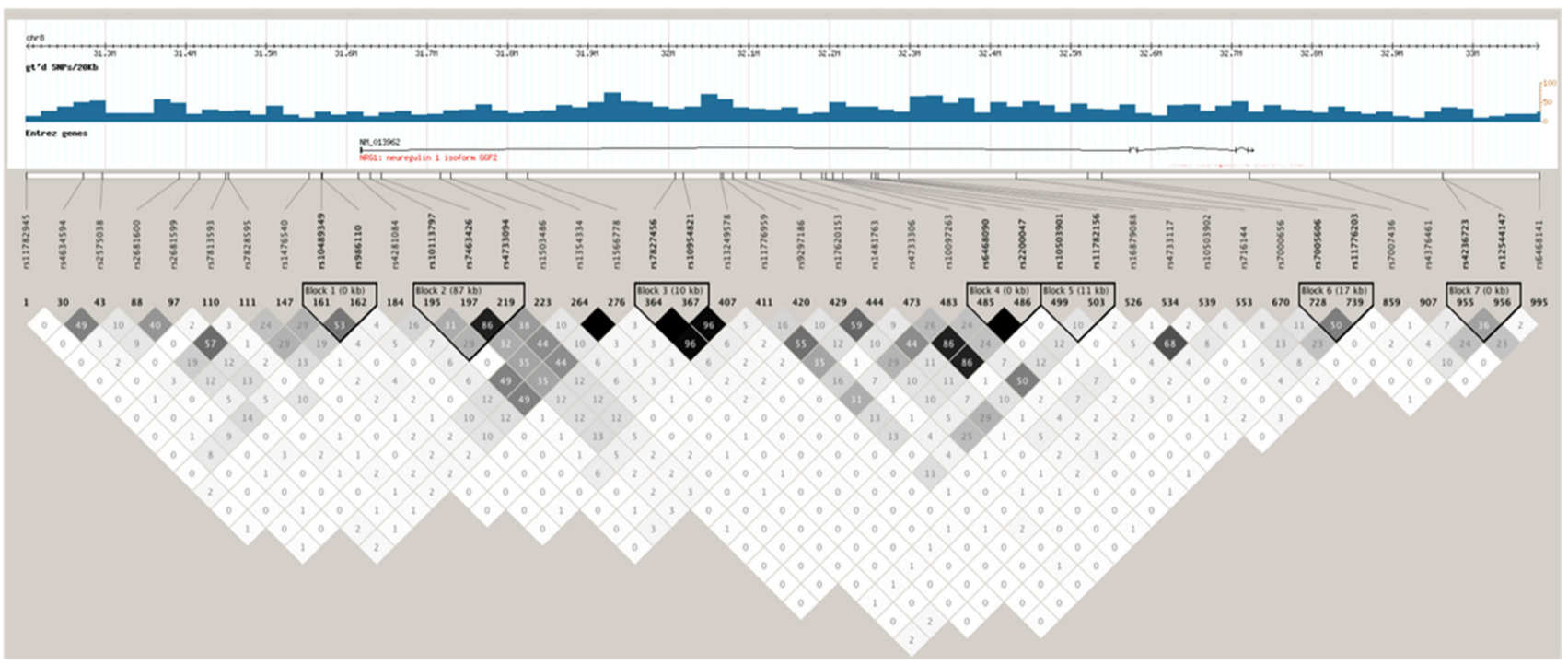

Fig. 2 LD patterns of NRG1 gene (Chr8:31,201,548..33,230,933) (Haploview 4.2, data from HapMap version 3, rel \#27, reference population CEU). Squares indicate $r^{2}$ values: $r^{2}=0$ : white square; $0<r^{2}<1$ : shades of gray squares; $r^{2}=1$ : black squares

end and more upstream regions of the gene. We speculate that variants clustering in this area could be correlated with positive symptoms. The second region contains SNPs with

Table 2 All ErbB4 SNPs that produced a nominal significant $p$ value $(<0.05$, before correction for multiple testing) with at least one PANSS item scores in the Aberdeen cohort

\begin{tabular}{|c|c|c|c|c|c|}
\hline \multirow[t]{2}{*}{ SNP } & \multirow[t]{2}{*}{ Allele } & \multicolumn{4}{|c|}{ PANSS psychopathological dimensions } \\
\hline & & Positive & Negative & General & Total \\
\hline rs4442936 & 2 & .0021 & .4262 & .0235 & .0185 \\
\hline rs7557116 & 2 & .0051 & .1474 & .0082 & .0066 \\
\hline rs6728697 & 2 & .0160 & .0090 & .0054 & .0017 \\
\hline rs1836729 & 1 & .0649 & .0268 & .1812 & .0327 \\
\hline rs1992027 & 1 & .9029 & .0186 & .8481 & .3487 \\
\hline rs1595068 & 2 & .0575 & .1465 & .0138 & .0190 \\
\hline rs1595067 & 2 & .0725 & .0150 & .0143 & .0065 \\
\hline rs6712652 & 1 & .0718 & .0445 & .0206 & .0139 \\
\hline rs953956 & 1 & .0223 & .3684 & .0955 & .0612 \\
\hline rs12619171 & 1 & .4981 & .2385 & .0346 & .0929 \\
\hline rs4130782 & 2 & .9952 & .0112 & .3832 & .1441 \\
\hline rs6722322 & 1 & .8286 & .0423 & .2590 & .1501 \\
\hline rs4672626 & 1 & .0295 & .6887 & .0838 & .0979 \\
\hline rs714393 & 1 & .1161 & .0402 & .6796 & .1206 \\
\hline rs1159709 & 1 & .5837 & .0461 & .8601 & .2746 \\
\hline rs9653337 & 2 & .0199 & .1789 & .1964 & .0575 \\
\hline rs1473636 & 1 & .7123 & .0402 & .0506 & .0607 \\
\hline rs7564926 & 2 & .0230 & .0524 & .0466 & .0140 \\
\hline rs1402766 & 1 & .0391 & .0666 & .1778 & .0405 \\
\hline rs1402769 & 1 & .0073 & .1554 & .3198 & .0563 \\
\hline rs10932428 & 1 & .0073 & .1554 & .3198 & .0563 \\
\hline
\end{tabular}

Significant $p$ values $(<0.05$, before correction for multiple testing) are highlighted in bold a nominal association with negative symptoms, clustering in a nearly $600-\mathrm{kb}$ long area and covering the gene length from its half toward its $3^{\prime}$ end. These findings might suggest that different portions of NRGl could be differentially implicated in the clinical presentation of schizophrenia or in the course of the illness. However, only a very limited number of studies used the symptom dimension approach with respect to NRG1 (Wilcox et al. 2002; Kendler et al. 2000), making comparison with our findings difficult. It is therefore difficult to establish whether the clustering of SNPs we found is unusual or unexpected. Unfortunately, none of the markers chosen as tagSNPs for this study matched any of the Icelandic core haplotype polymorphisms (Stefansson et al. 2002). This is due to the fact that our genotyping analyses derive from a previous comprehensive hypothesis-free genome-wide association study carried out through Illumina HumanHap genotyping BeadChips (Need et al. 2009). Our current analysis comprises the most strongly associated polymorphisms derived from the above-mentioned GWAS, and it is conceivable to assume that, for the considered loci, "the Illumina SNP sets did not include the best-associated variants from previous studies" (Need et al. 2009).

For the ErbB4 gene, we observed no difference in the distribution of nominally significant SNPs among psychopathological dimensions. Nevertheless, we found that a small cluster of variants spanning approximately $100 \mathrm{~kb}$ and covering the $3^{\prime}$ region of the gene had a tendency toward an association with negative and general psychopathological dimensions and the total score of symptoms. It would be interesting to establish if this particular site of ErbB4 is functionally involved in biological processes underling the phenotypic manifestations of schizophrenia, especially the 


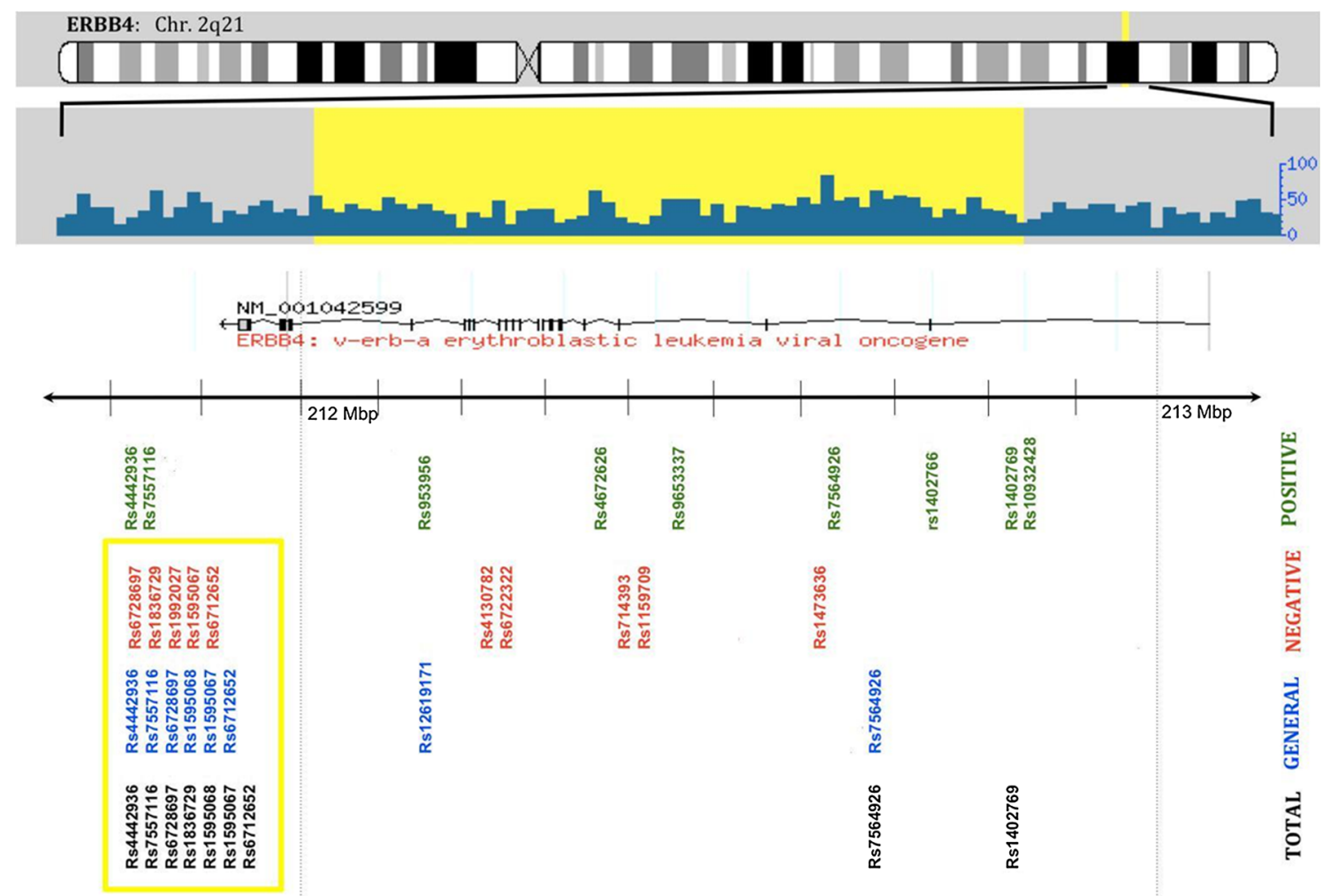

Fig. 3 ErbB4 locus along chr 2 and schematic representation of the SNPs that showed a positive nominal correlation with psychopathological symptoms categories (green positive dimension, red negative dimension, blue general dimension, black total dimension) (Color figure online)

negative and/or general psychopathology dimensions. The latter is just a speculative interpretation of the results, and in order to obtain a clearer picture of these neurobiological processes, further studies would be needed.

From the present study, it is also clear that some degree of LD exists among the SNPs tested, especially between adjacent variants. In general, both these genes are large and span multiple regions of $\mathrm{LD}\left(\mathrm{D}^{\prime}\right.$ measures in LD blocks in Figs. 2, 4). Hence, the clusters of SNPs we observed could point to a role for distinct genetic regions in the underlying biology of the symptoms investigated, suggesting that these variants might actually represent good proxies to real causative markers.

A recent and comprehensive review (Buonanno 2010) highlighted a major possible role for the NRG1/ErbB4 signaling pathway in the pathogenesis of schizophrenia. In fact, the ErbB4 is a post-synaptic receptor with a high expression in GABAergic neurons, and NRG1 is also involved in the regulation of GABAergic and dopaminergic pathways, as well as in the synaptic plasticity processes mediated by glutamate release (Buonanno 2010). It has been proposed that impairments in the latter pathways might be involved in the development of the cognitive, positive, and negative symptoms observed in schizophrenia
(Buonanno 2010). Within this hypothesis, only the cumulative impact of all the susceptibility variants investigated in this work would lead to the onset of psychotic symptoms, while considering each genetic polymorphism individually might lead to a loss of strength in association findings.

Finally, our hypothesis was first tested in the Aberdeen cohort and then we followed up the results in a second cohort recruited in Munich. Most of the SNPs clustering in the implicated regions were associated with schizophrenia in the Aberdeen sample, but no confirmation came from the Munich sample. This discrepancy might be due to either differences in clinical parameters, attributable to a different ascertainment at the two sites (Lelliott et al. 2010; Sederer 2010) or to real genetic differences between populations coming from different ancestries. Genetic differences between the Aberdeen and Munich populations were first assessed using the EIGENSTRAT software (Price et al. 2006). As a confirmation, the hidden population structure appeared to be low in the two populations. In fact, we calculated the Devlin and Roeder inflation factor $\lambda$ (Devlin and Roeder 1999), adjusting for the known Munich/Aberdeen split. We obtained a value of 1.013, indicating that stratification was very slight and did not significantly affect 


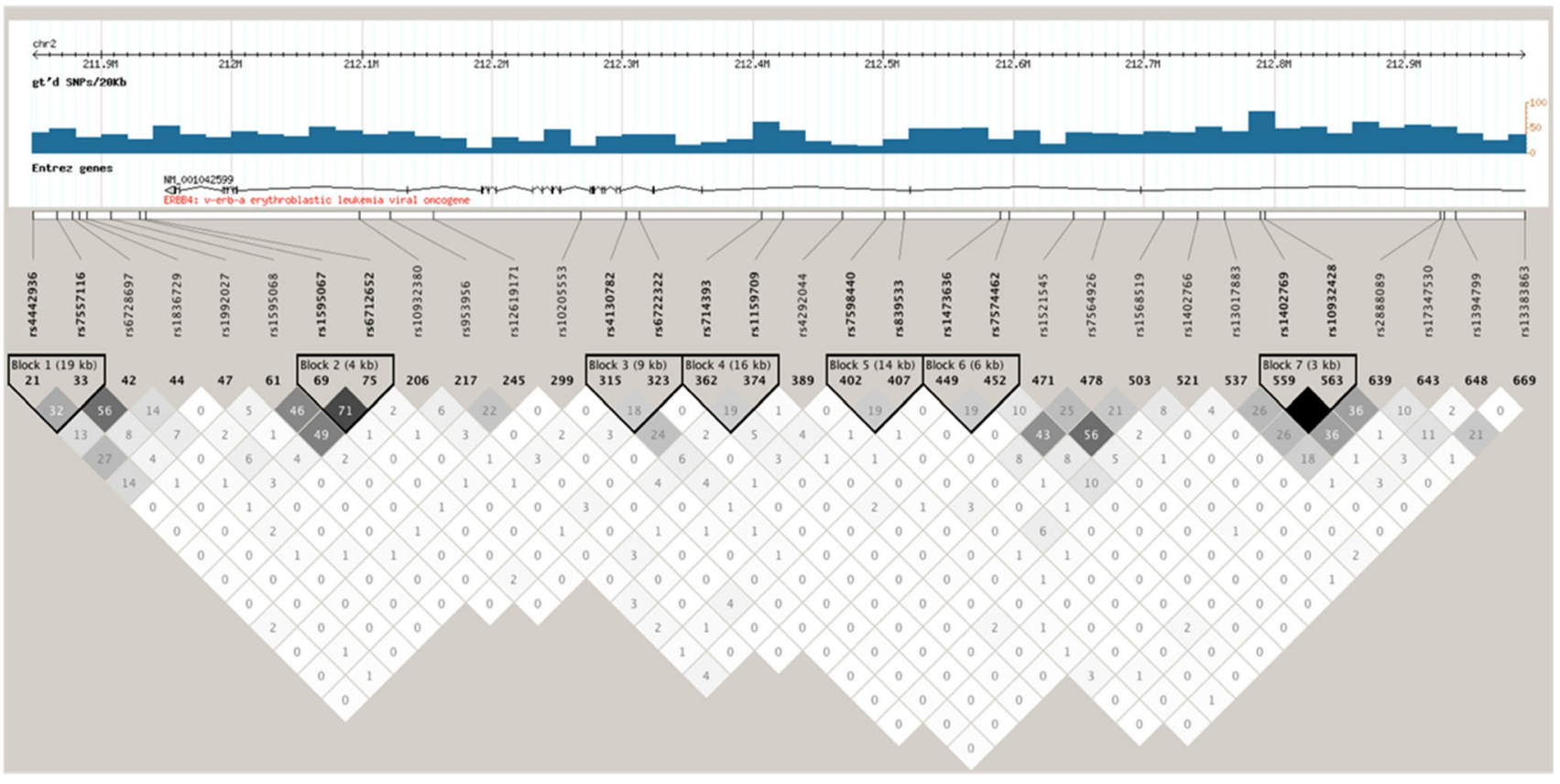

Fig. 4 LD patterns of ErbB4 gene (Chr2:211,847,782..212,992,266) (Haploview 4.2, data from HapMap version 3, rel \#27, reference population CEU). Squares indicate $r^{2}$ values: $r^{2}=0$ : white square; $0<r^{2}<1$ : shades of gray squares; $r^{2}=1$ : black squares

the detected genetic associations (Need et al. 2009). To our knowledge, the two populations are not phylogenetically different either. People from Scotland and Germany should share some common ancestors, tracing back to AngloSaxon invaders (Capelli et al. 2003). Moreover, neither Aberdeen nor Munich are known as recognizable geographical or genetic isolates. According to Novembre et al. (2008), geographically adjacent populations are typically very close from a genetic point of view, and they show a clear proximity between the Scotland and the German populations. An additional possible explanation for the lack of consistency in the nominal association signals between the Aberdeen and the Munich cohorts could be that these nominal associations have been detected by mere chance. For this reason, future confirmation studies are desirable in order to unravel any doubt about the validity of our results.

In conclusion, the difficulty in obtaining a consistent and clear-cut picture of the genetics of schizophrenia mirrors the marked clinical and neurobiological heterogeneity of the disorder. A comprehensive global model to capture the clinical heterogeneity in schizophrenia is still lacking. As long as we are not able to disentangle the question of heterogeneity at the clinical level, it is not likely that heterogeneity at the etiological and pathophysiological levels will be solved. Consequently, deconstructing schizophrenia into phenotypes based on symptoms, and then focusing on genes according to their biological function and putative involvement in this disorder, might be the best approach to overcome the lack of compelling genetic associations in schizophrenia genetic studies to date.
Acknowledgments We are grateful to the patients who participated in the study. We are indebted to the colleagues who contributed to the assessments. This study was supported by the Joint Projects 05 "Towards a new definition of schizophrenia: the biological basis of a core phenotype" with a grant to Mirella Ruggeri from GSK and University of Verona.

Conflict of interest None of the authors has a conflict of interest.

\section{References}

American Psychiatric Association (APA). (1994). Diagnostic and statistical manual of mental disorders (6th ed.,). Washington, DC: American Psychiatric Press.

Athanasiu, L., Mattingsdal, M., Kahler, A. K., Brown, A., Gustafsson, O., Agartz, I., et al. (2010). Gene variants associated with schizophrenia in a Norwegian genome-wide study are replicated in a large European cohort. Journal of Psychiatric Research, 44, $748-753$.

Bakker, S. C., Hoogendoorn, M. L., Hendriks, J., Verzijlbergen, K., Caron, S., Verduijn, W., et al. (2007). The PIP5K2A and RGS4 genes are differentially associated with deficit and non-deficit schizophrenia. Genes, Brain and Behavior, 6, 113-119.

Bakker, S. C., Hoogendoorn, M. L., Selten, J. P., Verduijn, W., Pearson, P. L., Sinke, R. J., et al. (2004). Neuregulin 1: Genetic support for schizophrenia subtypes. Molecular Psychiatry, 9, 1061-1063.

Benzel, I., Bansal, A., Browning, B. L., Galwey, N. W., Maycox, P. R., McGinnis, R., et al. (2007). Interactions among genes in the ErbB-Neuregulin signalling network are associated with increased susceptibility to schizophrenia. Behavioral and Brain Functions, 3, 31.

Bousman, C. A., Glatt, S. J., Chandler, S. D., Lohr, J., Kremen, W. S., Tsuang, M. T., \& Everall, I. P. (2013). Negative symptoms of psychosis correlate with gene expression of the $\mathrm{Wnt} / \beta$-catenin 
signaling pathway in peripheral blood. Psychiatry Journal 852930 .

Buonanno, A. (2010). The neuregulin signaling pathway and schizophrenia: From genes to synapses and neural circuits. Brain Research Bulletin, 83-84, 122-131.

Capelli, C., Redhead, N., Abernethy, J. K., Gratrix, F., Wilson, J. F., Moen, T., et al. (2003). A Y chromosome census of the British Isles. Current Biology, 13, 979-984.

Chen, P., Chen, J., Huang, K., Ji, W., Wang, T., Li, T., et al. (2011). Analysis of association between common SNPs in ErbB4 and bipolar affective disorder, major depressive disorder and schizophrenia in the Han Chinese population. Progress in NeuroPsychopharmacology and Biological Psychiatry, 36(1), 17-21.

Corvin, A. P., Morris, D. W., McGhee, K., Schwaiger, S., Scully, P., Quinn, J., et al. (2004). Confirmation and refinement of an 'at-risk' haplotype for schizophrenia suggests the EST cluster, Hs.97362, as a potential susceptibility gene at the Neuregulin-1 locus. Molecular psychiatry, 9, 208-213.

Crisafulli, C., Chiesa, A., Han, C., Lee, S. J., Park, M. H., Balzarro, B., Andrisano, C., Patkar, A. A., Pae, C. U., \& Serretti, A. (2012). Case-control association study for 10 genes in patients with schizophrenia: Influence of 5HTR1A variation rs10042486 on schizophrenia and response to antipsychotics. European Archives of Psychiatry and Clinical Neuroscience, 262(3), 199-205.

Davidson, L., \& McGlashan, T. H. (1997). The varied outcomes of schizophrenia. Canadian Journal of Psychiatry, 42, 34-43.

DeRosse, P., Malhotra, A. K., \& Lencz, T. (2012). Molecular genetics of the psychosis phenotype. Canadian Journal of Psychiatry, 57(7), 446-453.

Devlin, B., \& Roeder, K. (1999). Genomic control for association studies. Biometrics, 55, 997-1004

First, M. B., Spitzer, R. L., Gibbon, M., \& Williams, J. B. W. (1997). User's guide for the structured clinical interview for DSM-IV axis I disorders. Washington, DC: American Psychiatric Press.

Gong, Y. G., Wu, C. N., Xing, Q. H., Zhao, X. Z., Zhu, J., \& He, L. (2009). A two-method meta-analysis of Neuregulin 1(NRG1) association and heterogeneity in schizophrenia. Schizophrenia Research, 111, 109-114.

Hall, D., Gogos, J. A., \& Karayiorgou, M. (2004). The contribution of three strong candidate schizophrenia susceptibility genes in demographically distinct populations. Genes, Brain and Behavior, 3, 240-248.

Hegarty, J. D., Baldessarini, R. J., Tohen, M., Waternaux, C., \& Oepen, G. (1994). One hundred years of schizophrenia: A metaanalysis of the outcome literature. American Journal of Psychiatry, 151, 1409-1416.

Kay, S. R., Fiszbein, A., \& Opler, L. A. (1987). The positive and negative syndrome scale (PANSS) for schizophrenia. Schizophrenia Bulletin, 13, 261-276.

Kendler, K. S., Myers, J. M., O’Neill, F. A., Martin, R., Murphy, B., MacLean, C. J., et al. (2000). Clinical features of schizophrenia and linkage to chromosomes 5q, 6p, 8p, and $10 \mathrm{p}$ in the Irish study of high-density schizophrenia families. American Journal of Psychiatry, 157(3), 402-408.

Kim, J. H., Park, B. L., Pasaje, C. F., Bae, J. S., Park, C. S., Cha, B., Kim, B. J., Lee, M., Choi, W. H., Shin, T. M., Choi, I. G., Hwang, J., Koh, I., Woo, S. I., \& Shin, H. D. (2011). Lack of associations of Neuregulin 1 variations with schizophrenia and smooth pursuit eye movement abnormality in a Korean population. Journal of Molecular Neuroscience, 46(3), 476-482.

Kukshal, P., Bhatia, T., Bhagwat, A. M., Gur, R. E., Gur, R. C., Deshpande, S. N., et al. (2013). Association study of Neuregulin1 gene polymorphisms in a North Indian schizophrenia sample. Schizophrenia Research, 144(1-3), 24-30.
Lachman, H. M., Pedrosa, E., Nolan, K. A., Glass, M., Ye, K., \& Saito, T. (2006). Analysis of polymorphisms in AT-rich domains of neuregulin 1 gene in schizophrenia. American Journal of Medical Genetics Part B: Neuropsychiatric Genetics, 141B, 102-109.

Law, A. J., Kleinman, J. E., Weinberger, D. R., \& Weickert, C. S. (2007). Disease-associated intronic variants in the ErbB4 gene are related to altered ErbB4 splice-variant expression in the brain in schizophrenia. Human Molecular Genetics, 16, 129-141.

Lelliott, P., \& Bleksley, S. (2010). Improving the quality of acute inpatient care. Epidemiologia e Psichiatria Sociale, 9(4), 287-290.

Li, T., Stefansson, H., Gudfinnsson, E., Cai, G., Liu, X., Murray, R. M., et al. (2004). Identification of a novel neuregulin 1 at-risk haplotype in Han schizophrenia Chinese patients, but no association with the Icelandic/Scottish risk haplotype. Molecular Psychiatry, 9, 698-704.

Li, W., Zhou, N., Yu, Q., Li, X., Yu, Y., Sun, S., et al. (2013). Association of BDNF gene polymorphisms with schizophrenia and clinical symptoms in a Chinese population. American Journal of Medical Genetics Part B: Neuropsychiatric Genetics, 162B(6), 538-545.

Middle, F., Pritchard, A. L., Handoko, H., Haque, S., Holder, R., Bentham, P., et al. (2010). No association between neuregulin 1 and psychotic symptoms in Alzheimer's disease patients. Journal of Alzheimer's Disease, 20, 561-567.

Moon, R., Rollins, B., Mesén, A., Sequeira, A., Myers, R. M., Akil, H., et al. (2011). Lack of association to a NRG1 missense polymorphism in schizophrenia or bipolar disorder in a Costa Rican population. Schizophrenia Research, 131(1-3), 52-57.

Munafo, M. R., Attwood, A. S., \& Flint, J. (2008). Neuregulin 1 genotype and schizophrenia. Schizophrenia Bulletin, 34, 9-12.

Need, A. C., Ge, D., Weale, M. E., Maia, J., Feng, S., Heinzen, E. L., et al. (2009). A genome-wide investigation of SNPs and CNVs in schizophrenia. PLoS Genetics, 5, e1000373.

Nicodemus, K. K., Law, A. J., Radulescu, E., Luna, A., Kolachana, B., Vakkalanka, R., et al. (2010). Biological validation of increased schizophrenia risk with NRG1, ERBB4, and AKT1 epistasis via functional neuroimaging in healthy controls. Archives of General Psychiatry, 67(10), 991-1001.

Norton, N., Moskvina, V., Morris, D. W., Bray, N. J., Zammit, S., Williams, N. M., et al. (2006). Evidence that interaction between neuregulin 1 and its receptor erbB4 increases susceptibility to schizophrenia. American Journal of Medical Genetics Part B: Neuropsychiatric Genetics, 141B, 96-101.

Novembre, J., Johnson, T., Bryc, K., Kutalik, Z., Boyko, A. R., Auton, A., et al. (2008). Genes mirror geography within Europe. Nature, 456, 98-101.

Petryshen, T. L., Middleton, F. A., Kirby, A., Aldinger, K. A., Purcell, S., Tahl, A. R., et al. (2005). Support for involvement of neuregulin 1 in schizophrenia pathophysiology. Molecular Psychiatry, 10(366-374), 328.

Pitcher, G. M., Kalia, L. V., Ng, D., Goodfellow, N. M., Yee, K. T., Lambe, E. K., et al. (2011). Schizophrenia susceptibility pathway neuregulin 1-ErbB4 suppresses Src upregulation of NMDA receptors. Nature Medicine, 17(4), 470-478.

Price, A. L., Patterson, N. J., Plenge, R. M., Weinblatt, M. E., Shadick, N. A., \& Reich, D. (2006). Principal components analysis corrects for stratification in genome-wide association studies. Nature Genetics, 38, 904-909.

Purcell, S., Neale, B., Todd-Brown, K., Thomas, L., Ferreira, M. A., Bender, D., et al. (2007). PLINK: A tool set for whole-genome association and population-based linkage analyses. American Journal of Human Genetics, 81, 559-575.

Rethelyi, J. M., Bakker, S. C., Polgar, P., Czobor, P., Strengman, E., Pasztor, P. I., et al. (2010). Association study of NRG1, DTNBP1, RGS4, G72/G30, and PIP5K2A with schizophrenia 
and symptom severity in a Hungarian sample. American Journal of Medical Genetics Part B: Neuropsychiatric Genetics, 153B, 792-801.

Sederer, L. I. (2010). Inpatient psychiatry: Why do we need it? Epidemiologia e Psichiatria Sociale, 19(4), 291-295.

Shi, J., Levinson, D. F., Duan, J., Sanders, A. R., Zheng, Y., Pe'er, I., et al. (2009). Common variants on chromosome 6p22.1 are associated with schizophrenia. Nature, 460, 753-757.

Silberberg, G., Darvasi, A., Pinkas-Kramarski, R., \& Navon, R. (2006). The involvement of ErbB4 with schizophrenia: Association and expression studies. American Journal of Medical Genetics Part B: Neuropsychiatric Genetics, 141B, 142-148.

Stefansson, H., Sarginson, J., Kong, A., Yates, P., Steinthorsdottir, V., Gudfinnsson, E., et al. (2003). Association of neuregulin 1 with schizophrenia confirmed in a Scottish population. American Journal of Human Genetics, 72, 83-87.

Stefansson, H., Sigurdsson, E., Steinthorsdottir, V., Bjornsdottir, S., Sigmundsson, T., Ghosh, S., et al. (2002). Neuregulin 1 and susceptibility to schizophrenia. American Journal of Human Genetics, 71, 877-892.

Tao, R., Wei, J., Guo, Y., Yu, Y., Xu, Q., Shi, J., et al. (2006). The PLA2G4A gene and negative symptoms in a Chinese population. Schizophrenia Research, 86(1-3), 326-328.
Tosato, S., Dazzan, P., \& Collier, D. (2005). Association between the neuregulin 1 gene and schizophrenia: A systematic review. Schizophrenia Bulletin, 31, 613-617.

Tosato, S., \& Lasalvia, A. (2009). The contribution of epidemiology to defining the most appropriate approach to genetic research on schizophrenia. Epidemiologia e Psichiatria Sociale, 18(2), 81-90.

Van den Oord, E. J., Rujescu, D., Robles, J. R., Giegling, I., Birrell, C., Bukszar, J., et al. (2006). Factor structure and external validity of the PANSS revisited. Schizophrenia Research, 82, 213-223.

Wilcox, M. A., Faraone, S. V., Su, J., Van Eerdewegh, P., \& Tsuang, M. T. (2002). Genome scan of three quantitative traits in schizophrenia pedigrees. Biological Psychiatry, 52(9), 847-854.

Zhai, J., Yu, Q., Chen, M., Gao, Y., Zhang, Q., Li, J., et al. (2013). Association of the brain-derived neurotrophic factor gene G196A rs6265 polymorphisms and the cognitive function and clinical symptoms of schizophrenia. International Journal of Clinical and Experimental Pathology, 6(8), 1617-1623.

Zhao, X., Shi, Y., Tang, J., Tang, R., Yu, L., Gu, N., et al. (2004). A case control and family based association study of the neuregulin 1 gene and schizophrenia. Journal of Medical Genetics, 41, $31-34$. 